\title{
GENETIC RISK FACTORS FOR SPORADIC ALS
}

Numerous environmental and genetic factors have been implicated in sporadic amyotrophic lateral sclerosis (ALS); however, a detailed understanding of the genetic component of the disease has not yet been established. Michael van Es and colleagues have discovered two new sporadic ALS susceptibility loci one in a region that encodes a protein involved in the release of neurotransmitters such as glutamate, and another on chromosome 9p21.2 that has already been associated with familial ALS with frontotemporal dementia. These data support the hypothesis that genetic differences are important contributory factors in sporadic ALS and highlight the role of genes in this disease.

ALS is a neurodegenerative disease that is characterized by the progressive loss of motor neurons in the spinal cord and brain. The highly selective nature of the neuronal cell loss initially leads to muscle weakness and then, as the disease progresses, to muscle atrophy and paralysis. A number of genes are known to be associated with ALS; however, familial ALS only accounts for $5-10 \%$ of all cases. The underlining etiology of sporadic ALS is currently unknown and both environmental and genetic factors are suspected to be involved.

To investigate the involvement of genetic factors in sporadic ALS, van Es (University Medical Center Utrecht, Utrecht, The Netherlands) and colleagues performed a genome-wide association study among approximately 20,000 individuals divided between two independent cohorts. Single-nucleotide polymorphisms (SNPs) were initially identified using a high-throughput procedure, which utilized wholegenome SNP chips, in 2,323 patients with sporadic ALS and 9,013 control individuals. SNPs that were shown to be associated with ALS were then evaluated in a second, independent cohort of 2,532 patients with sporadic ALS and 5,940 control individuals.

Using this technique, van Es and colleagues found three SNPs that were shown to be positively associated with sporadic ALS. One of these SNPs is located in the UNC13A gene, "this gene is involved in vesicle priming for neurotransmission" notes van Es "[and] mice lacking this gene have severe muscle weakness." Furthermore, the protein encoded by the UNC13A gene has been found to be particularly important in glutamatergic synaptic transmission. This SNP is particularly interesting, as accumulating evidence indicates that glutamate excitotoxicity might be a contributory factor to motor neuron cell death in patients with ALS. The two other SNPs are located at chromosome 9p21.2 in a region that contains three genes-MOBKL2B, IFNK and C9orf72-and, as van Es explains, "the association locus in our study has also been identified in several large families with familial ALS [suggesting] that perhaps the same genes are involved in both [familial and sporadic ALS]."

Understanding how the genes identified in this study are associated with sporadic ALS is the next logical step. Furthermore, other ALS susceptibility loci are likely to exist, and these could be identified by larger genome-wide association studies. Previous genome-wide association studies have identified other candidate susceptibility genes for sporadic ALS, and a meta-analysis of the existing studies, might identify the most appropriate targets on which to base future therapies.

Nick Jones

Original article van Es, M. A. et al. Genome-wide association study identifies 19p13.3 (UNC13A) and 9p21.2 as susceptibility loci for sporadic amyotrophic lateral sclerosis. Nat. Genet. 41, 1083-1087 (2009). 